\title{
New Galactic Wolf-Rayet Stars Discovered via 2MASS + Spitzer/GLIMPSE
}

\author{
Jon C. Mauerhan ${ }^{1}$, Schuyler D. Van Dyk ${ }^{1}$, Pat W. Morris ${ }^{2}$ \\ ${ }^{1}$ Spitzer Science Center, IPAC, California Institute of Technology, M/C 220-6, Pasadena, CA \\ 91125, USA \\ email: mauerhan@ipac.caltech.edu \\ ${ }^{2}$ NASA Herschel Science Center, IPAC, California Institute of Technology, M/C 100-22, \\ Pasadena, CA 91125, USA
}

\begin{abstract}
We have undertaken a campaign to expand the census of WRs throughout the Galaxy using the 2MASS and Spitzer/GLIMPSE surveys. Free-free emission generated within the iondense winds of WRs generates a significant infrared excess which forms the basis of an excellent criterion for photometrically selecting WRs from the stellar field.
\end{abstract}

We report new results from our effort to identify obscured Wolf-Rayet stars (WRs) in the Galaxy. Candidates were selected by their near-infrared (2MASS (Cutri et al. 2003) and mid-infrared (Spitzer/GLIMPSE Churchwell et al. 2009) colour excesses, which are consistent with free-free emission from ionised stellar winds and thermal excess from hot dust. Mauerhan et al. (2009) have confirmed 12 new WRs in the Galactic disk, including $9 \mathrm{WN}$ type, and $3 \mathrm{WC}$; giving a total 27 discovered this way, including those in Hadfield et al. (2007). We classify one of the new stars as a possible dust-producing WC9d+OBI colliding-wind binary. A WC8 star in our sample appears to be a member of the stellar cluster Danks 1, in contrast to the rest of the confirmed Wolf-Rayet stars that generally do not appear to reside within dense stellar clusters. Therefore, the new WRs are either (1) runaways from clusters, (2) members of cluster which are confused with the background stellar field, or (3) formed in isolation. Follow-up observations to identify early-type sibling stars in the vicinity of the new WRs will elucidate their origins.

Based on the WR infrared colour selection criterion from Hadfield et al. (2007) and Mauerhan et al.(2009), the detection rate for WRs was $\approx 10 \%$. Recently, we have expanded our selection criteria to include more highly reddened sources, while excluding nearby contaminant stars more effectively (e. g., YSOs). This was made possible in part by colour-magnitude restrictions, and the addition of a new colour selection sieve based on [8]-[24] $\mu \mathrm{m}$ colours from the Spitzer/MIPSGAL survey (Carey et al. 2009). In addition, we have also cross-correlated our latest candidate WR sample with archival Chandra and $X M M$ point-source catalogues. With these new refinements, the most recent observations indicate that our WR detection rate has improved dramatically; 30 new WRs have been discovered, $1 / 3$ of which are serendipitous X-ray detections.

\section{References}

Carey, S. J. et al. 2009, PASP, 121, 76

Churchwell, E. et al. 2009, PASP, 121, 213

Cutri, R. M. et al. 2003, "Explanatory Supplement to the 2MASS All Sky Data Release"

Hadfield, L. J., van Dyk, S. D., Morris, P. W. et al. 2007, MNRAS, 376, 248

Mauerhan, J. C., van Dyk, S. D., \& Morris, P. W. 2009, PASP, 121, 591 